\title{
Altered Adiposity after Extremely Preterm Birth
}

\author{
SABITA UTHAYA, E. LOUISE THOMAS, GAVIN HAMILTON, CAROLINE J. DORÉ, \\ JIMMY BELL, AND NEENA MODI \\ Division of Paediatrics, Obstetrics and Gynaecology [S.U., N.M.], Faculty of Medicine, Imperial College \\ London, Chelsea \& Westminster Hospital, London SW10 9NH, UK, Molecular Imaging Group [E.L.T., \\ J.B.], MRI Unit, Imaging Sciences Department [G.H.], MRC Clinical Sciences Centre, London W12 ONN, \\ UK, MRC Clinical Trials Unit [C.J.D.], London NW1 2DA, UK
}

\begin{abstract}
ABST
The quantity and distribution of adipose tissue are markers of
morbidity risk. The third trimester of human development is a
period of rapid adipose tissue deposition. Preterm infants may be
at risk of altered adiposity. We measured anthropometric indices
and quantified total, subcutaneous, and intraabdominal adipose
tissue volumes using whole-body magnetic resonance adipose
tissue imaging in 38 infants born at <32 wk gestational age,
when they reached term, and 29 term-born infants. The preterm
infants at term were significantly lighter and shorter than the
term-born infants, but there was no significant difference in head
circumference SD score or total adiposity. The preterm infants
had a highly significant decrease in subcutaneous adipose tissue
and significantly increased intraabdominal adipose tissue. Accel-
erated postnatal weight gain was accompanied by increased total
and subcutaneous adiposity. Illness severity was the principal
determinant of increased intraabdominal adiposity. Our data
provide evidence of causal pathways linking accelerated postna-
tal growth with increased total and subcutaneous adiposity, and
illness severity with altered adipose tissue partitioning. We sug-
gest that these observations may in part explain the associations
between small size at birth and later disease. Preterm infants may
be at risk in later life of metabolic complications through in-
creased and aberrant adiposity. (Pediatr Res 57: 211-215, 2005)
\end{abstract}
The distribution, as well as the quantity of AT, is a marker of morbidity risk. Adipose tissue is metabolically active, and differences in adiposity-associated morbidity are in keeping with the known depot-specific differences in the expression of genes coding for adipocyte proteins (1). In adults, subcutaneous adiposity is primarily related to circulating leptin and generalized obesity, whereas intraabdominal (visceral) adiposity is associated with insulin resistance, even in lean individuals, and with dyslipidemia (2). Such associations are increas-

Received June 25, 2004; accepted July 28, 2004.

Correspondence: Neena Modi, MB.ChB, MD, FRCP, FRCPCM, Division of Paediatrics, Obstetrics \& Gynaecology, Faculty of Medicine, Imperial College London, Chelsea \& Westminster Hospital, 369 Fulham Rd., London SW10 9NH, UK; e-mail: n.modi@imperial.ac.uk

Supported by a grant from the Medical Research Council London, UK.

DOI: 10.1203/01.PDR.0000148284.58934.1C
AT, adipose tissue

\%ATM, percentage adipose tissue mass

ATV, adipose tissue volume

GA, gestational age

IAIAT, intraabdominal internal adipose tissue

\%IAIATV, percentage intra abdominal internal adipose tissue volume

IAT, internal adipose tissue

\%IATV, percentage internal adipose tissue volume

NAIAT, nonabdominal internal adipose tissue

\%NAIATV, percentage nonabdominal internal adipose tissue volume

OFC, occipitofrontal circumference

SCAT, subcutaneous adipose tissue

\%SCATV, percentage subcutaneous adipose tissue volume

SDS, standard deviation score

SDSG, standard deviation score gain

TAT, total adipose tissue

\%level 1\&2, percentage level 1 and 2 intensive care ingly reported in children, but the age at which abnormal patterns of adiposity are established is not known $(3,4)$. The third trimester of pregnancy is a period of rapid AT deposition. Infants born extremely preterm are profoundly deficient in AT. Subsequently, their postnatal course is often marked by prolonged nutritional compromise, chronic illness, and poor growth. These are plausible determinants of both reduced and aberrant AT deposition.

Previous in vivo body composition studies in infants have been indirect and therefore unable to quantify specific AT depots. We have developed the application of whole-body magnetic resonance (MR) AT imaging to newborn infants (5) and have published data on adipose distribution in a separate cohort of term infants (6). The technique is noninvasive and radiation free and enables direct quantification of individual AT compartments. 
The aim of this study was to address the null hypothesis that total adiposity and AT distribution in extremely preterm infants, upon reaching the age of term-equivalent, does not differ from term-born newborns. Secondary aims were to examine growth performance in the preterm infants at age termequivalent and the effects of size at birth, postnatal growth, postnatal diet, and illness severity upon total adiposity and AT distribution.

\section{METHODS}

Infants were recruited from the Hammersmith and Queen Charlotte's Hospital neonatal unit and postnatal wards between December 2002 and December 2003. The study was approved by the local research ethics committee and written parental consent was obtained. Preterm infants born at $<32$ wk gestation were studied at age term-equivalent. Healthy, appropriately grown term infants were studied within $6 \mathrm{~d}$ of birth.

Magnetic resonance imaging and image analysis. Infants underwent whole-body magnetic resonance imaging as previously described by our group (5). This was performed in natural sleep with the infant positioned supine. Heart rate and oxygen saturation are monitored continuously using pulse oximetry. A neonatologist was present throughout the procedure.

MR images were acquired on a Phillips 1.5 Tesla system using a rapid T1-weighted spin-echo sequence (repetition time of $600 \mathrm{~ms}$, echo time of 16 $\mathrm{ms})$. The serial isocenter technique was used, in which the infant was moved through the magnet on a mobile platform for full-body imaging. Images were obtained with a slice and interslice thickness of $5 \mathrm{~mm}$. There is little effect on the overall quantification of ATV if gaps between slices do not exceed one slice thickness (7). Images (Fig. 1) were analyzed by a single observer (S.U.) using a commercially available software program (SliceOMatic, Version 4.2, Tomovision, Montreal, Canada) that has been widely used in body composition studies. Initially a filter was used to distinguish between different graylevel regions on each slice. The observer then used an interactive slice editor program to verify and, where necessary, edit the segmentation.

Classification of AT compartments. The classification of AT in previously published imaging studies in adults and children is inconsistent and the definition of intraabdominal or visceral AT has often not been clear (8). Methods to quantify AT have also varied, with some studies using a singleslice technique and others restricting imaging to the abdomen. There is further controversy over what constitutes the so-called "deep subcutaneous abdominal" depot. This distinct compartment is clearly separated from subcutaneous AT by a fascial plane. It is morphologically and metabolically different from subcutaneous AT and behaves like intraabdominal AT in that it is a risk factor for insulin resistance $(9,10)$. For the purposes of this study, we defined AT as either subcutaneous (SCAT) or internal (IAT). We further classified IAT as either intraabdominal (IAIAT) or nonabdominal (NAIAT). We defined the intraabdominal compartment as IAT contained in the slices ranging from the top of the liver to the heads of the femurs inside the fascial plane referred to above. NAIAT was predominantly located in the head and neck.

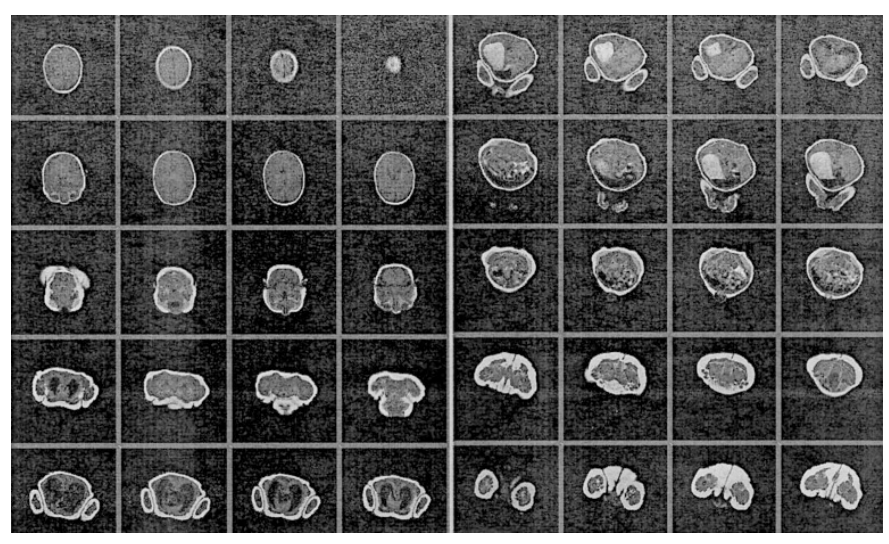

Figure 1. MR images showing subcutaneous and internal AT.
Calculation of AT area, volume, and mass. Tissue area $\left(\mathrm{cm}^{2}\right)$ for each slice was calculated as the sum of the pixels multiplied by pixel area. Tissue volume $\left(\mathrm{cm}^{3}\right)$ for each slice was calculated by multiplying the tissue area by the sum of the slice thickness $(0.5 \mathrm{~cm})$ and the interslice distance $(0.5 \mathrm{~cm})$. ATV was converted to mass based on a density of AT of $0.9 \mathrm{~g} / \mathrm{cm}^{3}$ (11). Therefore, ATV (liters) $\times 0.9=$ AT mass $(\mathrm{kg})$.

We expressed total AT mass as a percentage of infant weight (\%ATM) and individual compartments as percentage of total ATV (\%SCATV, \%IATV, \%IAIATV, \% NAIATV $)$. Thus, total ATV $=($ SCATV + IATV $)$, where IATV $=($ IAIATV + NAIATV $)$.

We determined intraobserver variability separately for the preterm and the term infants. The coefficient of variation in the preterm infants was $2.8 \%$ for TAT, $0.77 \%$ for SCAT, and $10.56 \%$ for IAIAT and, for the term infants, $1.33 \%, 0.39 \%$, and $1.45 \%$, respectively. This compares favorably with our previous work and that reported in the adult literature where the coefficient of variation for SCAT is in the range of $2-5 \%$ and that for IAIAT from $9 \%$ to $18 \%(9,12,13)$.

Sample size. We aimed to study a minimum of 27 infants in each group within the 1-y study period as, based upon our previous data, this would allow us to detect a $3 \%$ difference in total adiposity ( $80 \%$ power, $5 \%$ significance) (6).

Anthropometry. Weight and OFC at birth were documented and, at the time of imaging, weight, OFC, and length were measured by a single observer (S.U.). Scales used were accurate to $0.2 \mathrm{~g}$ (Marsden Professional Baby Scale, London, UK), length was measured with a Rollametre (Raven Equipment Ltd., Dunmow, Essex, UK), and OFC with a nondistensible tape measure (Child Growth Foundation, London, UK).

Nutritional data. The type of intravenous nutrition (10\% dextrose or parenteral nutrition) and enteral feed (donor-banked expressed breast milk, maternal expressed breast milk, preterm formula, term formula) the preterm babies received was noted for every day from birth to the day before imaging. The number of days of breast milk received (taking into account that babies might be on more than one type of nutrition on any given day) was expressed as a percentage of the total number of days from birth to the day before imaging (\% breast milk).

Illness severity. We documented the number of days of level 1 and 2 intensive care received using the British Association of Perinatal Medicine criteria (2001). Data required to assign level of care were captured daily and defined the clinical status of the infant. Level 1 is the most intensive, and level 4 is normal care of a healthy newborn baby. We expressed days of level 1 and 2 intensive care as a percentage of the number of days from birth to the day before imaging (\%level 1\&2 IC).

Postnatal growth. We expressed weight, length, and OFC as SDS adjusted for age and gender. In the preterm group, rate of growth between birth and age term-equivalent was expressed as weight SDS gain (weight SDSG) and OFC SDSG (Child Growth Foundation). This is the difference in SDS adjusted for sex and reference correlations between measurements at two time points (14).

Statistical analysis. Data were analyzed using SPSS Version 12 (SPSS Inc., Chicago, IL) and Stata Release 8 (StataCorp, College Station, TX). The preterm and term data were compared using the independent samples $t$ test. Equal variances were not assumed because of the greater variability in the preterm data. Data obtained at the time of imaging was adjusted to the overall mean age at imaging using separate robust regression lines for each group. Unless otherwise stated, data are presented as mean (SD). Linear and multiple regression analyses were used to examine the influence of GA at birth, percentage breast milk received, \%level 1\&2 IC, and weight and head circumference SDSG on \%ATM, \%SCATV, and \%IAIATV. When analyzing the effect of SDSG, the SDS at birth was taken into account to allow for regression to the mean.

\section{RESULTS}

We studied 67 infants (Table 1). Thirty-eight were born preterm (14 male, 24 female) and 29 at term (12 male, 17 female). At birth, mean (SD) GA, weight, and head circumference were as follows: preterm, $28.8 \mathrm{wk}$ (2.1 wk); term, 39.9 wk (1.4 wk); preterm, $1.19 \mathrm{~kg}(0.37 \mathrm{~kg})$; term, $3.47 \mathrm{~kg}(0.29$ 
Table 1. Comparison of preterm infants at age term-equivalent and term-born infants; anthropometry and absolute values of adipose tissue

\begin{tabular}{lcrrr}
\hline & $\begin{array}{c}\text { Preterm } n=38 \\
{[\text { mean (SD)] }}\end{array}$ & $\begin{array}{c}\text { Term } n=29 \\
{[\text { mean (SD)] }}\end{array}$ & $p$ & $95 \%$ CI for difference \\
\hline Postmenstrual age at imaging (wk) & $38.57(1.66)$ & $40.17(1.47)$ & $<0.001$ & $0.83,2.37$ \\
Weight SDS & $-1.15(1.14)$ & $-0.55(0.74)$ & 0.012 & $0.14,1.06$ \\
OFC SDS & $-0.11(1.41)$ & $0.22(1.08)$ & 0.29 & $-0.28,0.94$ \\
Length SDS & $-1.19(1.42)$ & $0.59(0.82)$ & $<0.001$ & $1.22,2.33$ \\
Total adipose tissue (1) $\dagger$ & $0.557(0.230)$ & $0.661(0.108)$ & 0.017 & $0.019,0.189$ \\
SCAT (1) $\dagger$ & $0.490(0.215)$ & $0.609(0.102)$ & 0.004 & $0.040,0.198$ \\
IAIAT (1) $\dagger$ & $0.026(0.011)$ & $0.021(0.005)$ & 0.012 & $-0.009,-0.001$ \\
\hline
\end{tabular}

$\dagger$ Adjusted to overall mean age at imaging.

$\mathrm{kg})$; and preterm, $26.3 \mathrm{~cm}(2.3 \mathrm{~cm})$, term, $34.9 \mathrm{~cm}(1.3 \mathrm{~cm})$, respectively.

Thirty-four of the preterm infants had received antenatal steroids. None of the infants had received postnatal steroid therapy. The preterm infants received breast milk ranging from $9 \%$ to $96 \%$ of days between birth and term (median, $89 \%$ ) and required level $1 \& 2$ IC for 0 to $88 \%$ of days (median, 29\%).

In the preterm group, median weight SDSG to age termequivalent was -1.24 , with a range of -3.57 to 1.86 . In contrast, median OFC SDSG was 0.34, with a range of -4.47 to 3.53. Mean (SD) weight, length, and $\mathrm{OFC}$ of the preterm babies at term-equivalent were $2.68(0.26) \mathrm{kg}, 47.06(2.93) \mathrm{cm}$, and $33.79(1.8) \mathrm{cm}$, respectively. Weight and length SDS of the preterm babies at age term-equivalent were significantly less than the term born babies but there was no significant difference in OFC SDS (Table 1).

Actual postmenstrual age at imaging of the preterm and term infants differed [preterm, $38.6 \mathrm{wk}$ (1.7 wk); term, $40.2 \mathrm{wk}$ (1.5 wk); $p<0.001]$. Absolute ATV were therefore compared after adjusting for age at imaging (Table 1). Absolute total and subcutaneous ATV were significantly lower in the preterm infants, but there was a significant increase in absolute IAIATV [mean difference, $0.005 \mathrm{~L} ; 95 \%$ confidence interval (CI), -0.009 to $-0.001 ; p=0.01]$.

Although significantly smaller than term infants, \%ATM in the preterm infants at term was not significantly different from the term-born infants (Table 2). This masked a highly significant difference in the distribution of AT. Subcutaneous AT (\%SCATV) was reduced in the preterm babies (preterm, $88.5 \%$; term $91.9 \%$; $95 \%$ CI for difference, 2.36 to $4.42 ; p<$ 0.001 ) and intraabdominal IAT (\%IAIATV) was increased (preterm, $4.62 \%$; term, $3.11 \%$; 95\% CI for difference, -1.92 to $-1.10 ; p<0.001)$.

In the preterm infants, linear regression analysis demonstrated the adverse impact of increasing \%level $1 \& 2$ IC on $\%$ SCATV $(r=-0.58, p<0.001)$, weight SDSG $(r=-0.50$, $p=0.002)$ and OFC SDSG $(r=-0.33, p=0.042)$.
Weight SDSG, but not OFC SDSG, showed a significant positive correlation with \% ATM $(r=0.40, p=0.014)$. Weight SDSG was also significantly correlated with \%SCATV $(r=$ 0.404, $p=0.012$ ).

$\%$ SCATV increased with increasing GA at birth $(r=0.39$, $p=0.016$ ). There was a negative correlation between birth weight SDS and birth OFC SDS and \%IAIATV, although this failed to reach significance $(r=-0.31, p=0.056 ; r=-0.29$, $p=0.074$, respectively).

The negative impact of increased \%level $1 \& 2$ IC on $\%$ SCATV was confirmed in a multiple regression analysis (Table 3), allowing for GA at birth, birth weight SDS, and weight SDSG (adjusted $R^{2} 33.7 \%, B=-0.076, \mathrm{SE}=0.03, p$ $=0.017$ ). Conversely, a multiple regression model also incorporating GA at birth, birth weight SDS, and weight SDSG showed increased \%IAIATV with increasing \%level 1\&2 IC (adjusted $R^{2} 20.7 \%, B=0.033, \mathrm{SE}=0.012, p=0.009$ ).

We found no evidence of any effect of breast milk, nor of gender, on AT outcomes.

\section{DISCUSSION}

We have made the novel observation that AT distribution is altered in the preterm infant at term with significantly increased intraabdominal adiposity. The mean excess IAIAT in the preterm infants was $5 \mathrm{~cm}^{3}$ when expressed as an absolute volume and $1.51 \%$ when expressed as a percentage of total ATV. Is this likely to be of clinical relevance? To our knowledge, there have been no studies in infants and few studies in adults that have measured whole-body AT and expressed IAIAT as a percentage of total ATV. Thomas et al. (7), in a study of 67 women with a range of body mass indices, found percentage of IAIAT varied from a mean of $5.7 \%$ in women with a low body mass index to $8.7 \%$ in those considered obese. In children and adults, increased intraabdominal adiposity is a recognized marker for insulin resistance, dyslipidemia, and other components of the metabolic syndrome. Weight loss confers improve-

Table 2. Percentage adiposity and adipose tissue distribution, preterm infants at age term-equivalent and term-born infants

\begin{tabular}{lcccc}
\hline & $\begin{array}{c}\text { Perm } n=29 \\
{[\text { mean (SD)] }}\end{array}$ & $p$ & $p$ & $95 \%$ CI for difference \\
\hline [mean (SD)] & $17.0(4.0)$ & $18.3(2.5)$ & 0.64 & $(-1.23,1.97)$ \\
\% SCATV & $88.5(2.9)$ & $91.9(1.1)$ & $<0.001$ & $(2.36,4.42)$ \\
\% IAIATV & $4.62(1.03)$ & $3.11(0.64)$ & $<0.001$ & $(-1.92,-1.10)$ \\
\hline
\end{tabular}


Table 3. Multiple regression models for determinants of adipose tissue distribution in preterm infants

\begin{tabular}{|c|c|c|c|c|c|}
\hline Dependent variable & Model adjusted $R^{2}$ & Predictors & Unstandardized coefficient & SE & $p$ \\
\hline \multirow[t]{3}{*}{ \%SCATV } & $33.7 \%$ & GA at birth & -0.446 & 0.373 & 0.24 \\
\hline & & Birth weight SDS & 0.435 & 0.437 & 0.33 \\
\hline & & Weight SDSG & 0.454 & 0.414 & 0.28 \\
\hline \multirow[t]{3}{*}{ \%IAIATV } & $20.7 \%$ & GA at birth & 0.424 & 0.146 & 0.006 \\
\hline & & Birth weight SDS & -0.156 & 0.171 & 0.37 \\
\hline & & Weight SDSG & -0.051 & 0.162 & 0.75 \\
\hline
\end{tabular}

ment in insulin resistance, but it is the concomitant decrease in IAIAT that appears to be important (15). IAIAT accounts for about $18 \%$ of total body AT in aging rats, and surgical removal results in reversal of insulin resistance and improved glucose tolerance (16). In otherwise healthy adults, excessive intraabdominal adiposity tends to accompany generalized obesity but in certain pathologic states, such as human immunodeficiency virus-associated lipodystrophy and other acquired and congenital lipodystrophies, intraabdominal adiposity occurs without an increase in subcutaneous or total adiposity (17-19). In either situation, a decrease in IAIAT is a therapeutic goal and confers clinical benefit, suggesting that an altered distribution, with a relative increase in IAIAT, carries the same risk as an absolute increase in IAIAT (20). The preterm infants in our study had both a relative (expressed as a percentage of total ATV) and an absolute increase (despite their significantly smaller body size) in IAIAT, suggesting that this is a real effect that is likely to be clinically relevant.

What might determine this increase in intraabdominal adiposity? Excessive glucocorticoid exposure, whether exogenous as in steroid treated subjects, or endogenous as in Cushing's disease, results in IAIAT accumulation. Stress-associated glucocorticoid release is thought to be one of the mechanisms underlying the metabolic syndrome. We have observed elevated glucocorticoid levels in preterm infants in intensive care (unpublished data). In the present study, we have shown a strong association between disease severity and the quantity of IAIAT. We have also shown that antenatal compromise, as represented by low birth weight SDS and birth OFC SDS, was negatively correlated with \%IAIATV, although this did not reach statistical significance. These observations suggest that exposure to an adverse environment either in utero, or after birth, increases intraabdominal adiposity. Neonatal steroid treatment has been shown to decrease insulin sensitivity in small-for-gestational-age infants (21). None of the infants in our study received postnatal steroid therapy, but chronic or excessive endogenous glucocorticoid exposure might underlie the increase in intraabdominal adiposity, as well as the suppression in linear growth.

Nutritional support after preterm birth has largely been directed toward mimicking intrauterine growth and after intrauterine growth restriction, in achieving catch-up through accelerated growth because poor growth, particularly poor head growth, is associated with adverse neurodevelopmental outcome. However, concern has recently been raised about the promotion of rapid weight gain in infancy. Singhal et al. (22), in a post hoc analysis of infants recruited in the early 1980 s into a series of randomized controlled trials of infant feeding, showed that markers of insulin resistance were greater in a group of preterm infants fed a nutrient-enriched formula in comparison with preterm babies fed a nutrient-poor intake in whom there had been no rapid postnatal growth. These authors conclude that a period of relative nutrient deprivation in the postnatal period may be advantageous. Our data suggest an alternative hypothesis explaining the association between preterm birth and insulin resistance, namely illness severity, shown by us to be the principal determinant of increased intraabdominal adiposity.

Poor growth frequently accompanies extremely preterm birth and clinical management generally aims to promote nutritional intake. The preterm babies in this study were lighter and shorter at term-equivalent than the term-born infants. Head growth, however, appeared to have been maintained and, despite being smaller, percentage adiposity in the preterm group was similar to that of the term-born babies. This suggests that the poor linear and somatic growth in the preterm group was unlikely to have been due to poor nutrition.

Rapid postnatal growth has been associated with obesity in childhood and there has been considerable debate about the protective effect of breast milk (23-25). Dewey (26), in a review of published evidence, concluded that although a protective effect of breast milk remains plausible, the magnitude of any such effect is likely to be small. Clinical practice in our unit is to use maternal or banked expressed breast milk in the first few weeks after birth. We found no evidence of a relationship between quantity of breast milk received and total, intraabdominal, or subcutaneous AT.

Epidemiologic research indicates that small birth size and accelerated postnatal growth are risk factors for obesity in childhood and adult life but causal pathways are unknown (27) and direct observational data are lacking. We found a significant correlation between accelerated postnatal growth, expressed as weight SDSG, and total adiposity (\%ATM). This suggests that by increasing adiposity, accelerated growth may increase susceptibility to other late-onset superimposed determinants of obesity. We regret that we do not have data on linear growth, as the finding of a similar relationship between length SDSG and adiposity would strengthen this conclusion.

Associations have also been described between small size at birth and later-life risks of hypertension, dyslipidemia, and insulin resistance (28). Preterm birth may be associated with risks that are distinct from those of poor intrauterine growth, although these are likely to overlap. We have previously shown that antenatal growth restriction is associated with a marked 
reduction in subcutaneous, but relative conservation of intraabdominal AT (6). Failure to distinguish between intrauterine growth restriction and preterm birth may, at least in part, explain the heterogeneity in clinical phenotype in childhood and adult life in infants who were small at birth. Our data suggest possible explanations for the associations between preterm birth and hence small birth size, with later-life disease, namely accelerated postnatal growth, resulting in increased total and subcutaneous adiposity, and illness severity, resulting in altered AT partitioning. It will now be important to establish whether infants born extremely preterm manifest an altered metabolic profile and are at risk of later overt disease. Therapeutic targets might prove to be the attenuation of the endogenous glucocorticoid response to neonatal intensive care and the restriction of rapid somatic and linear growth.

Acknowledgments. The authors thank the babies and their parents for participating in this study.

\section{REFERENCES}

1. Vidal H 2001 Gene expression in visceral and subcutaneous adipose tissues. Ann Med 33:547-555

2. Wajchenberg BL, Giannella-Neto D, da Silva ME, Santos RF 2002 Depot-specific hormonal characteristics of subcutaneous and visceral adipose tissue and their relation to the metabolic syndrome. Horm Metab Res 34:616-621

3. Goran MI, Bergman RN, Gower BA 2001 Influence of total vs. visceral fat on insulin action and secretion in African American and white children. Obes Res 9:423-431

4. Weiss R, Dufour S, Taksali SE, Tamborlane WV, Petersen KF, Bonadonna RC, Boselli L, Barbetta G, Allen K, Rife F, Savoye M, Dziura J, Sherwin R, Shulman GI, Caprio S 2003 Prediabetes in obese youth: a syndrome of impaired glucose tolerance, severe insulin resistance, and altered myocellular and abdominal fat partitioning. Lancet 362:951-957

5. Harrington TA, Thomas EL, Modi N, Frost G, Coutts GA, Bell JD 2002 Fast and reproducible method for the direct quantitation of adipose tissue in newborn infants. Lipids 37:95-100

6. Harrington TA, Thomas EL, Modi N, Frost G, Bell JD 2004 Distribution of adipose tissue in the newborn. Pediatr Res 55:437-441

7. Thomas EL, Saeed N, Hajnal JV, Brynes A, Goldstone AP, Frost G, Bell JD 1998 Magnetic resonance imaging of total body fat. J Appl Physiol 85:1778-1785

8. Shen W, Wang Z, Punyanita M, Lei J, Sinav A, Kral JG, Imielinska C, Ross R, Heymsfield SB 2003 Adipose tissue quantification by imaging methods: a proposed classification. Obes Res 11:5-16

9. Kelley DE, Thaete FL, Troost F, Huwe T, Goodpaster BH 2000 Subdivisions of subcutaneous abdominal adipose tissue and insulin resistance. Am J Physiol Endocrinol Metab 278:E941-E948

10. Smith SR, Lovejoy JC, Greenway F, Ryan D, deJonge L, de la Bretonne J, Volafova J, Bray GA 2001 Contributions of total body fat, abdominal subcutaneous adipose tissue compartments, and visceral adipose tissue to the metabolic complications of obesity. Metabolism 50:425-435

11. Ross R, Leger L, Guardo R, De Guise J, Pike BG 1991 Adipose tissue volume measured by magnetic resonance imaging and computerized tomography in rats. J App Physiol 70:2164-2172

12. Elbers JM, Haumann G, Asscheman H, Seidell JC, Gooren LJ 1997 Reproducibility of fat area measurements in young, non-obese subjects by computerized analysis of magnetic resonance images. Int J Obes Relat Metab Disord 21:1121-1129

13. Staten MA, Totty WG, Kohrt WM 1989 Measurement of fat distribution by magnetic resonance imaging. Invest Radiol 24:345-349

14. Cole TJ, Freeman JV, Preece MA 1998 British 1990 growth reference centiles for weight, height, body mass index and head circumference fitted by maximum penalized likelihood. Stat Med 17:407-429

15. Goodpaster BH, Kelley DE, Wing RR, Meier A, Thaete FL 1999 Effects of weight loss on regional fat distribution and insulin sensitivity in obesity. Diabetes 48:839847

16. Gabriely I, Ma XH, Yang XM, Atzmon G, Rajala MW, Berg AH, Scherer P, Rossetti L, Barzilai N 2002 Removal of visceral fat prevents insulin resistance and glucose intolerance of aging: an adipokine-mediated process? Diabetes 51:2951-2958

17. Agarwal AK, Simha V, Oral EA, Moran SA, Gorden P, O'Rahilly S, Zaidi Z, Gurakan F, Arslanian SA, Klar A, Ricker A, White NH, Bindl L, Herbst K, Kennel K, Patel SB, Al-Gazali L, Garg A 2003 Phenotypic and genetic heterogeneity in congenital generalized lipodystrophy. J Clin Endocrinol Metab 88:4840-4847

18. Saint-Marc T, Partisani M, Poizot-Martin I, Rouviere O, Bruno F, Avellaneda R, Lang JM, Gastaut JA, Touraine JL 2000 Fat distribution evaluated by computed tomography and metabolic abnormalities in patients undergoing antiretroviral therapy: preliminary results of the LIPOCO study. AIDS 14:37-49

19. Simha V, Garg A 2003 Phenotypic heterogeneity in body fat distribution in patients with congenital generalized lipodystrophy caused by mutations in the AGPAT2 or seipin genes. J Clin Endocrinol Metab 88:5433-5437

20. Thoni GJ, Fedou C, Brun JF, Fabre J, Renard E, Reynes J, Varray A, Mercier J 2002 Reduction of fat accumulation and lipid disorders by individualized light aerobic training in human immunodeficiency virus infected patients with lipodystrophy and/or dyslipidemia. Diabetes Metab 28:397-404

21. Leipala JA, Raivio KO, Sarnesto A, Panteleon A, Fellman V 2002 Intrauterine growth restriction and postnatal steroid treatment effects on insulin sensitivity in preterm neonates. J Pediatr 141:472-476

22. Singhal A, Fewtrell M, Cole TJ, Lucas A 2003 Low nutrient intake and early growth for later insulin resistance in adolescents born preterm. Lancet 361:1089-1097

23. Ong KK, Preece MA, Emmett PM, Ahmed ML, Dunger DB, ALSPAC Study Team 2002 Size at birth and early childhood growth in relation to maternal smoking, parity and infant breast-feeding: longitudinal birth cohort study and analysis. Pediatr Res 52:863-867

24. Cianfarani S, Germani D, Branca F 1999 Low birthweight and adult insulin resistance: the "catch-up growth" hypothesis. Arch Dis Child Fetal Neonatal Ed 81:F71F73

25. Eriksson JG, Forsen T, Tuomilehto J, Winter PD, Osmond C, Barker DJ 1999 Catch-up growth in childhood and death from coronary heart disease: longitudinal study. BMJ 318:427-431

26. Dewey KG 2003 Is breastfeeding protective against child obesity? J Hum Lact 19:9-18

27. Ong KK, Ahmed ML, Emmett PM, Preece MA, Dunger DB 2000 Association between postnatal catch-up growth and obesity in childhood: prospective cohort study. BMJ 320:967-971

28. Barker DJ, Winter PD, Osmond C, Margetts B, Simmonds SJ 1989 Weight in infancy and death from ischaemic heart disease. Lancet 2:577-580 